\title{
Case \\ Report \\ Adenocarcinoma at angle of treitz: A report of two cases with review of literature
}

\author{
Koli Pushpa, Dewoolkar VV, Butale U \\ Department of General Surgery, K.J. Somaiya Hospital and Research Centre, Everad Nagar, Mumbai, \\ India.
}

Correspondence to: Dr. V.V. Dewoolkar, E-mail: dewoolkarmedicine@gmail.com

\begin{abstract}
We report two patients with adenocarcinoma at angle of treitz who presented with upper GI(gastrointestinal) obstruction and underwent segmental resection of duodeno-jejunal junction. Preoperative investigations failed to reveal the nature of the lesion suggesting the elusive nature of these lesions, importance of clinical suspicion and decision-making.
\end{abstract}

Key words: Adenocarcinoma, angle of treitz, duodenojejunal growth

\section{Introduction}

Primary malignancies of small bowel are rare and present a unique challenge in terms of preoperative diagnosis, treatment decisions and postoperative management. Adenocarcinoma occurring at ligament of treitz are extremely rare and difficult to diagnose but have a better prognosis compared to duodenal adenocarcinoma. Segmental resection is the treatment of choice but the effect of postoperative chemotherapy is not clear from literature, however it is worth giving.

\section{Case Reports}

\section{Case 1}

A 73-year-old female patient was admitted twice in year 2005 with upper abdominal pain and vomiting of three and a half months duration which responded to conservative treatment. The patient got readmitted for the third time after a month with copious bilious vomiting and abdominal pain which was suggestive of upper GI obstruction. Patient was evaluated with abdominal ultrasound, upper GI endoscopy and CT scan of abdomen. The ultrasound and CT scan were essentially normal. D uring the endoscopy the scope was passed till $3^{\text {rd }}$ part of duodenum and was normal. After a brief period of fasting and nasogastric aspiration for three days the patient did not improve. A small bowel obstruction was suspected and patient was posted for operation. On exploration there was a $3.5 \mathrm{~cm}$ growth at duodeno-jejunal junction with enlarged mesenteric lymph nodes, without involvement of adjacent structures. Segmental resection of duodenojejunal (D-J) junction with growth with $1.5 \mathrm{~cm}$ of margin and clearance of loco regional lymphnodes was done. Postoperative recovery was uneventful. The histopathology confirmed it as moderately differentiated adenocarcinoma, reaching the serosa with no lymphovascular invasion. Patient refused postoperative chemotherapy and did not come for regular follow-up.

After one and half years of initial surgery patient got readmitted with features of gastric outlet obstruction and a palpable epigatric mass. U Itrasound revealed multiple mesenteric lymph nodes with minimal ascites. On exploration there were multiple lymph nodes at the root of mesentery; the largest was measuring $10 \times 8$ $\mathrm{cm}$. The lymph node mass was compressing the pylorus and distal common bile duct causing obstruction. There were no bowel lesions and liver metastasis. A palliative bypass in the form of anterior gastrojejunostomy, cholecystojejunostomy and jejunojejunostomy was done. Patient recovered well and survived for a further period of six months.

\section{Case 2}

A 40-year-old male patient was admitted with complaints of upper abdominal pain, bilious vomiting and constipation for one and half months. $\mathrm{He}$ was hepatitis B surface antigen positive (HBSAg). On upper $G$ I endoscopy there was suspicion of superior 
mesenteric artery syndrome(SM A syndrome), as the duodenum was dilated till the third part, beyond which scope could not be negotiated Though ultrasound abdomen was normal, CT scan suggested the possibility of SM A Syndrome, hence the patient was explored.

On exploration the duodenum was dilated and there was a growth at ligament of treitz measuring $4 \mathrm{~cm}$ without involvement of serosa or enlarged lymph nodes [Figure 1]. There was no ascites or liver metastases. Duodenojejunal segmentectomy with $1 \mathrm{~cm}$ margin and duodenojejunal anastomosis was done. Postoperatively the patient recovered well and histopathology suggested moderately differentiated adenocarcinoma [Figure 2] which was reaching serosa without perineural/ lymphovascular invasion. Patient was given chemotherapy and at six months of follow-up is doing well.

\section{Discussion}

Small bowel cancers are a rare entity. The small bowel

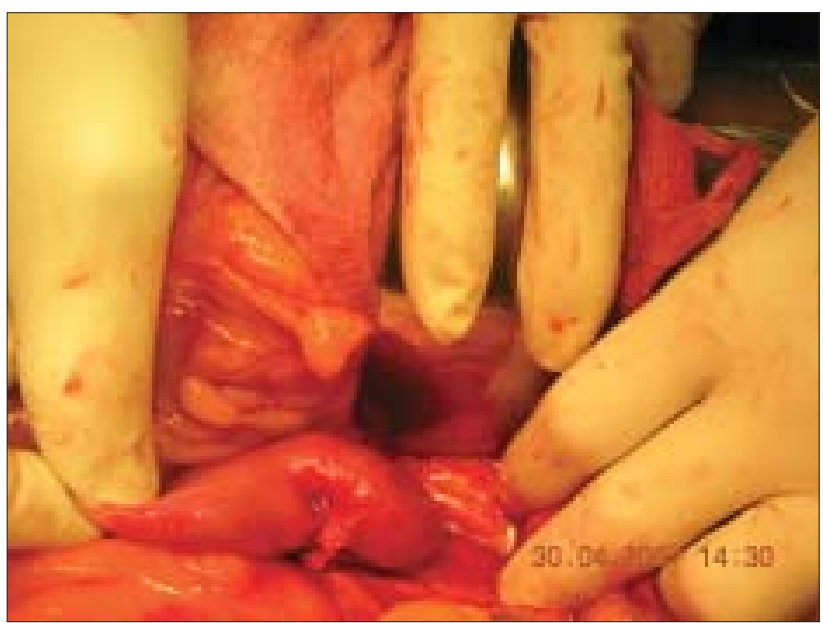

Figure 1: Operative photograph showing growth at angle of treitz after mobilisation

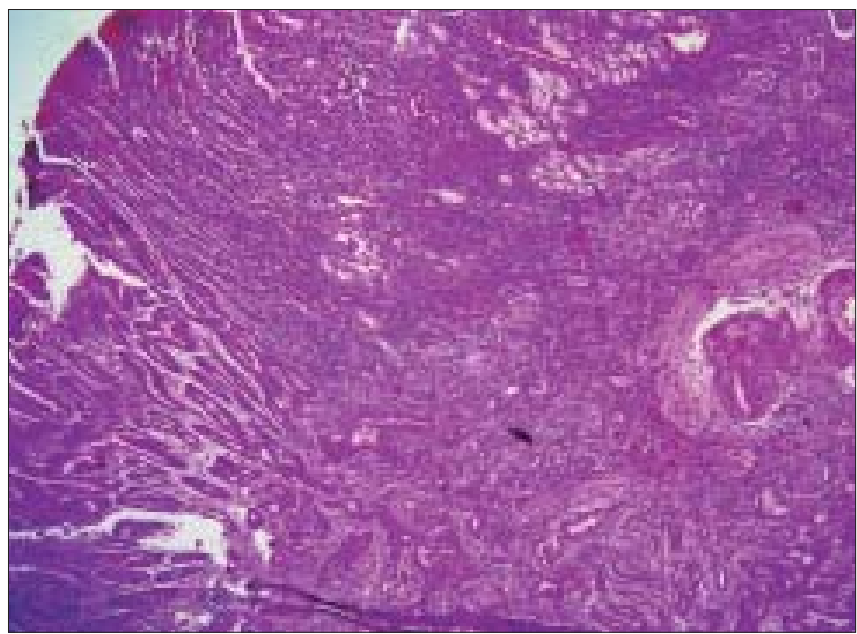

Figure 2: Photomicrograph showing tumor arising from mucosa (adjacent to brunners glands) and infiltrating muscularis propria comprises $80 \%$ of the length of gastrointestinal tract, yet it counts for only $1 \%$ of all GIT malignancies. True incidence of adenocarcinomas occurring at ligament of treitz is unknown, but duodenal adenocarcinomas constitute about 0.3 to $0.4 \%$ of all GIT cancers.

Adenocarcinomas usually present in the 6th and 7th decades. In a study of 129 patients with primary small bowel cancers by Tocchi et al, ${ }^{[1]} 33 \%$ were adenocarcinomas, $29 \%$ were carcinoids and $19 \%$ were lymphomas. H alf of all adenocarcinomas occur in the duodenum, nearly $20 \%$ in jejunum and a little over $10 \%$ in ileum and $14 \%$ occur in unspecified sites. ${ }^{[2]}$

Among the duodenal carcinomas two third occur in the periampullary region. $\mathrm{R}$ isk factors that are associated with small bowel adenocarcinoma include familial adenomatous polyposis coli, Gardners and Turcots syndrome, Crohns disease, Coeliac disease, Lynch syndrome, immunosuppression. etc., however, both of our patients did not have any predisposing factors. The second patient was only HBSAg positive.

The extreme rarity and vagueness of presenting symptoms makes it hard to diagnose these tumors. ${ }^{[1,2]}$ The most common presenting complaint is intermittent pain due to partial intestinal obstruction. $5-10 \%$ of the patients may present with manifestations of hemorrhage like malena and anemia. Other features include anorexia and weight loss. The mean duration of symptoms before presentation is 10 months (range, 0-24 months). ${ }^{[3]}$

There are no specific methods to diagnose these tumors in an early stage. Most of the cases require special investigations for diagnosis. Barium contrast has a diagnostic accuracy of $83 \% .^{[1,2]}$. Enteroclysis is the investigation of choice for the small bowel intrinsic pathology. Enteroscopy by the "push" method using the pediatric colonoscope can yield a biopsy or a "sonde" enteroscopy can be used to view the lesion. Capsule endoscopy and double balloon enteroscopy (DBE) are the newer diagnostic modalities. DBE was described by Yamamoto et al I in 2001, which helps in diagnosing the small bowel lesions along with biopsy taking. ${ }^{[4]}$

Duodenal first and second part tumors are treated by Whipples' procedure. For resectable cancers of third and fourth part of duodenum, segmental resection is the treatment of choice. Duodenojejunal segmentectomy is the treatment of choice for angle of treitz tumors with lymph node clearance, though lymph node positivity does not preclude resection, ${ }^{[5]}$ and the prognosis of these tumors is good. ${ }^{[6]}$ In both of our cases segmental resection with lymph node clearance in the first case was done. Large tumor size, transmural invasion and 
moderate to poor tumor grade were negatively correlated with survival. Lymph node status did not influence survival, whereas in a multivariate analysis of 101 patients, nodal metastases, positive margins, and stage were significant prognostic predictors. The three and five-year actuarial survival rates of patients with negative nodes were $73 \%$ and $68 \%$, respectively, compared with $35 \%$ and $22 \%$ in patients with nodal metastases. ${ }^{[7]}$ Jejunal and proximal ileal malignancies require resection and anastomosis while distal ileal ones require a right hemicolectomy. The role of adjuvant chemotherapy and radiotherapy in small bowel adenocarcinoma is not clearly defined and it has been found in some studies that adjuvant treatment after complete resection has not been shown to diminish the risk for subsequent recurrence. ${ }^{[3]}$

The overall 5 -year survival is 17 to $25 \%,{ }^{[2]}$ and median survival is 19.7 months, ${ }^{[1]}$ prognosis being worse in duodenal malignancies. Factors affecting outcome are tumor diameter, histological grade and serosal involvement. Patients should be followed-up closely because the possibility of developing another abdominal carcinoma is high. As associated polyps are nearly always duodenal or jejunal, a preoperative or intraoperative endoscopy of the upper gastrointestinal tract including the initial portion of the jejunum should be done to detect their presence so as to reduce the risk of an early recurrence.

\section{References}

1. Tocchi A, Mazzoni G, Puma F, Miccini M, Cassini D, Bettelli E, et al. Adenocarcinoma the third and fourth portions of the duodenum: Results of surgical treatment. Arch Surg 2003; 138:80-5.

2. Howe JR, Karnell LH, Menck HR, Scott-Conner C. Adenocarcinoma of the small bowel: Review of the National Cancer Data Base, 19851995. Cancer 1999;86:2693-706.

3. Talamonti MS, Goetz LH, Rao S, Joehl RJ. Primary cancers of the small bowel: Analysis of prognostic factors and results of surgical management. Arch Surg 2002; 137:564-70

4. Yamamoto H, Kita H, Sunada K, Hayashi Y, Sato H, Yano T, et al. Clinical outcomes of double-balloon endoscopy for the diagnosis and treatment of small-intestinal diseases. Clin Gastroenterol Hepatol 2004;2:1010-6.

5. Rose DM, Hochwald SN, Klimstra DS, Brennan MF. Primary duodenal adenocarcinoma: A ten-year experience with 79 patients. J Am Coll Surg 1996; 183:89-96.

6. Carloni A, Perri S, Gola P, Lotti R, Caterino G, Altilia F, et al. Adenocarcinoma of duodenojejunal flexure: A report of 2 clinical cases and a review of the literature. Ann Ital Chir 2000;71:133-8.

7. Bakaeen FG, Murr MM, Sarr MG, Thompson GB, Farnell MB, Nagorney $\mathrm{DM}$, et al. What prognostic factors are important in duodenal adenocarcinoma? Arch Surg 2000; 135:635-42.

Source of Support: Nil, Conflict of Interest: None declared. 